\title{
On the role of stiffness design for fingertip trajectories of underactuated modular soft hands
}

This is the peer reviewed version of the following article:

Original:

Hussain, I., Salvietti, G., Malvezzi, M., Prattichizzo, D. (2017). On the role of stiffness design for fingertip trajectories of underactuated modular soft hands. In Proceedings - IEEE International Conference on Robotics and Automation (pp.3096-3101). New York : IEEE [10.1109/ICRA.2017.7989354].

Availability:

This version is availablehttp://hdl.handle.net/11365/1012986

since 2017-09-13T15:49:48Z

Publisher:

IEEE

Published:

DOI:10.1109/ICRA.2017.7989354

Terms of use:

Open Access

The terms and conditions for the reuse of this version of the manuscript are specified in the publishing policy. Works made available under a Creative Commons license can be used according to the terms and conditions of said license.

For all terms of use and more information see the publisher's website.

(Article begins on next page) 


\title{
On the Role of Stiffness Design for Fingertip Trajectories of Underactuated Modular Soft Hands
}

\author{
Irfan Hussain ${ }^{1}$, Gionata Salvietti ${ }^{1,2}$, Monica Malvezzi ${ }^{1,2}$ and Domenico Prattichizzo ${ }^{1,2}$
}

\begin{abstract}
In this work, we propose a method to compute the stiffness of flexible joints and its realization in order to let the fingers track a certain predefined trajectory. We refer to tendon-driven, underactuated and passively compliant hands composed of deformable joints and rigid links. Specific stiffness and pre-form shapes can be assigned to the finger joints can be given $s$ such that a single-cable actuation can be used. We firstly define a procedure to determine suitable joints stiffness and then we propose a possible realization of soft joints using rapid prototyping techniques. The stiffness computation is obtained leveraging on the the mechanics of tendon-driven hands and on compliant systems, while for its implementation beam theory has been exploited. We validate the proposed framework both in simulation and with experiments using the robotic SoftSixthFinger, a wearable robot for grasping compensation in patients with a paretic hand, as a case study. The proposed framework can be used to design the stiffness of the passive joints in several model of underactuated tendon-driven soft hands so to improve their grasping capabilities.
\end{abstract}

\section{INTRODUCTION}

Robotic grasping and manipulation require an elevated level of complexity which involves not only the mechanical structures of the end effectors, but also comprise their capability to exploit their interaction with the environment. Very sophisticated hand with a high number of degrees of freedom have been developed to mostly resemble the kinematic structure of the human hand. However, these complex devices resulted poorly capable to interact with objects and with the environment to achieve a proposed task. This also favored the development of formal mathematical approaches [1] and planning strategies [2] that consider the environment surrounding the object to grasp as an obstacle to avoid. In addition, the presence of many actuators decreases the robustness of the devices and demand for a complex control system able to manage the high number of control inputs. To deal with the above mentioned issues, a novel generation of underactuated compliant hands is growing in the robotic community in the last years. Differently from classical multifingered hand, in this soft hands the interaction with the environment and the simplification of the actuation and the control is central to the device design. Underactuated hands have desirable adaptability to shapes, and can be effectively

The research leading to these results has received funding from the EU Horizon 2020/2015 project No 645599 "SoMa" and from EU Horizon 2020/2015 project No 688857 "SOFTPRO”.

${ }^{1}$ Università degli Studi di Siena, Dipartimento di Ingegneria dell'Informazione, Via Roma 56, 53100 Siena, Italy. [irfan.hussain] @unisi.it [salviettigio, malvezzi, prattichizzo] daism.unisi.it

${ }^{2}$ Department of Advanced Robotics, Istituto Italiano di Tecnologia, Via Morego 30, 16163 Genoa, Italy. implemented using relatively simple differential and elastic elements [3]. The transmission solutions allow motion of other joints to continue after contact occurs on a coupled link [4]. Passive adaptability consent to drive the device with a reduced number of control parameters. The devices can endure collisions with hard objects and even strikes from a hammer without breaking into pieces. Reasons for adding passive elements are manifold, including storing elastic energy, avoiding tendon slackness, passive compliance, the distribution of forces over a large contact area and ensuring the uniqueness of the position [5]. Shape adaptation increases the grasp performance by compensating the uncertainties in sensing and actuation so to help stabilizing the grasp [6]. Remarkable examples of underactuated compliant hands are, for instance, the Pisa/IIT hand [5], the RBO Hand [7] and the SDM Hand [8].

Most of the work in literature focuses on the development of new devices proposing different solutions in terms of actuation and materials for hand realization. Few works are instead focusing on systematic ways to improve the dexterity of new soft hands [9]. If you consider a highly underactuated soft hand (e.g., a five fingered hand with 15 DoFs and one motor with a tendon driven actuation) there are few parameters to be tuned in order to design a desired motion for the robotic fingers. If the kinematic and the actuation system is fixed, a possible way to design a desired fingertip trajectory is to opportunely tune the stiffness of the passively compliant joints. In this work, we present a procedure to compute the stiffness ratio between the passive compliant joints so to obtain a desired trajectory for the fingers. The proposed method assumes a given target motion of the fingertips and a given maximum actuation force for the tendon driven system to compute the stiffness value of the passive joints. We propose a modular approach to define robotic hands. In most of the solutions existing in the literature, the hand is composed of a series of identical fingers, and the modularity is exploited at the finger level, (eg., see [10]). In the approach we propose, each phalanx, composed of a flexible and a stiff part, is modular. Beside the number and position of fingers in the hand, we can furthermore change the number of phalanges and some of their structural properties similar to what proposed in [11].

The problem that we want to solve is: how can we design finger joint stiffness so that, when applying a certain force to the tendons, the joint configuration vector $\mathbf{q}$ assumes a desired shape $\mathbf{q}_{r}$ and thus the fingertip follows a desired trajectory? To address this problem we propose a method to compute the stiffness given a certain kinematics of the 
robotic hand. We then leverage on beam theory to find a way to compute the stiffness for a particular geometry of the soft parts. We demonstrate the accuracy of the generated trajectory in a case study represented by Soft-SixthFinger [12]. The possibility of selecting the hand pre-shaping and the finger flexion trajectory are important to enhance the soft hand grasping capabilities [13].

The rest of the paper is organized as follows. In Section II the proposed mathematical framework is described in details. Section III describes all the simulations and the experiments carried out with the Soft-SixthFinger as a case study for underactuated compliant grippers. Finally, in Section IV conclusion and future work are outlined.

\section{Fingertip tRAJECTORY DEFINITION THROUGH JOINT STIFFNESS DESIGN}

In this section, we propose a general mathematical framework to compute the stiffness of compliant joints necessary to track a desired fingertip flexion trajectory. In particular, we firstly define how to compute the stiffness values and later how to realize passive compliant joints with a given stiffness once the geometry of the passive joints is defined.

\section{A. Problem definition}

Let us assume a hand composed of $n_{f}$ fingers, with $n_{q}$ joints, actuated by a series of $n_{t}$ tendons. For the sake of simplicity we assume that all the joints in the hand are revolute (R), so that the variable $q_{i}$ describing $i$-th displacement is a rotation. Let us indicate with $\mathbf{q}=\left[q_{1}, \cdot, q_{n_{q}}\right]^{\mathrm{T}} \in \Re^{n_{q}}$ a vector containing hand joint rotations and with $\mathbf{t} \in \Re^{n_{t}}$ tendon displacements.

From the kinematic analysis of hand fingers, it is possible to relate tendon displacements $\mathbf{t}$ to hand joint configuration $\mathrm{q}$ as

$$
\mathbf{t}=\mathbf{T q},
$$

where $\mathbf{T} \in \Re^{n_{t} \times n_{q}}$ is a transformation matrix whose elements depends on finger pulleys' sizes and tendon routing topology and is independent from hand posture [3]. By applying the Principle of Virtual Work to the hand it is possible to obtain the dual static relationship

$$
\boldsymbol{\tau}=\mathbf{T}^{\mathrm{T}} \mathbf{f}
$$

where $\boldsymbol{\tau} \in \Re^{n_{q}}$ represent hand joint torques and $\mathbf{f} \in \Re^{n_{t}}$ is a vector containing tendons' pulling forces. If the hand is free and the joints have passive elastic elements, the following relationship between joint torques and hand posture can be set

$$
\boldsymbol{\tau}+\mathbf{K}_{q} \Delta \mathbf{q}=\mathbf{0},
$$

where $\mathbf{K}_{q} \in \Re^{n_{q} \times n_{q}}$ is joint stiffness matrix, symmetric and positive definite, and $\Delta \mathbf{q}$ indicates a configuration variation evaluated w.r.t. a reference (rest) position of the hand $\mathbf{q}_{0}$, i.e., $\Delta \mathbf{q}=\mathbf{q}-\mathbf{q}_{0}$. For the sake of simplicity let us assume $\mathbf{q}_{0}=\mathbf{0}$.

If $\mathbf{K}_{q}$ matrix is diagonal, i.e. if the joints are independent, the problem is straightforward. Eq. (3) can be rewritten, in this case, as

$$
\boldsymbol{\tau}+\mathbf{Q k}_{q}=\mathbf{0},
$$

where $\mathbf{Q} \in \Re^{n_{q} \times n_{q}}$ is defined as $\mathbf{Q}=\operatorname{diag}(\mathbf{q})$, while $\mathbf{k}_{q} \in$ $\Re^{n_{q}}$ is a vector collecting joint stiffness. Taking into account eq. (2), the system can be solved as follows

$$
\mathbf{k}_{q}=\mathbf{Q}_{r}^{-1} \mathbf{T}^{\mathrm{T}} \mathbf{f}_{r} .
$$

The solution is a vector containing hand joint stiffness values that allows to obtain a configuration $\mathbf{q}_{r}$ of the hand when the tendons are pulled with a force $\mathbf{f}_{r}$. Normalizing vector $\mathbf{k}_{q}$ we obtain a base for the subspace of possible stiffness combinations that can be used to track a desired trajectory. In fact, the trajectory shape depends on the stiffness ratios between two adjacent joints, rather than on their actual value. The final value of $\mathbf{k}_{q}$ depends on the characteristic of the used material and on the geometry used for the compliant joints as better detailed in Sec. III.

\section{B. Modular hand modeling}

Let us consider a generic joint $i$ on the finger $j$ of the hand. Let us indicate with $l$ the length of the elastic element that constitute the joint. According to the modular approach proposed in this paper, we assume that this value is the same for all the joints, however the following model can be easily extended to hands with different joints. Let us indicate with $h$ the distance between the elastic element bending axis and the tendon when the joint is in its rest position. Also this value is the same for all the joints, according to the modular structure of the proposed hand design. When the tendon on the $j$-th finger is pulled the elastic elements in the joints will bend: let us suppose that the joint $i$ is rotated by an angle $q_{i}=\theta_{i}$, the corresponding variation of tendon length in the joint is indicated with $\Delta l_{i}$. We suppose that the bending elastic element assumes a circular arc shape, and assuming small values of $\theta$, so that we can approximate $\left(\sin \frac{\theta}{2}\right) \approx \frac{\theta}{2}$, we have

$$
\Delta l_{i} \approx h_{i} \theta_{i}
$$

If the $j$-th finger of the hand is composed of $n_{q_{j}}$ modules, its overall displacement $t_{j}$ is given by

$$
t_{j}=\sum_{i=1}^{n_{q_{j}}} \Delta l_{i}=\mathbf{h}_{j} \mathbf{q}_{j}
$$

where $\mathbf{h}_{j}=\left[h_{1}, \cdots, h_{n_{q j}}\right] \in \Re^{n_{q j}}$. Applying the same procedure to all the fingers we can evaluate the tendon transformation matrix $\mathbf{T} \in \Re^{n_{f} \times n_{q}}$ as

$$
\mathbf{T}=\left[\begin{array}{cccc}
\mathbf{h}_{1} & \mathbf{0} & \cdots & \mathbf{0} \\
\mathbf{0} & \mathbf{h}_{2} & \cdots & \mathbf{0} \\
\cdots & \cdots & \cdots & \cdots \\
\mathbf{0} & \mathbf{0} & \cdots & \mathbf{h}_{n_{f}}
\end{array}\right]
$$

\section{Passive joint design}

Given the stiffness of each joint of robotic finger in order to follow a certain trajectory, the next step is its realization in robotic fingers hardware structure. The stiffness depends on the design (geometry) and material properties of the module parts. Thus, we need to evaluate the relationship between the geometric and material properties of modules to the desired stiffness ratio for each phalange of soft finger. 
In this paper, we refer to a modular approach in the design: the basic idea is to realize modules composed of a flexible passive joint and a rigid link which can be combined together to build underactuated soft robotic fingers.

When the actuator applies a force $f_{r j}$ through the $j$-th tendon, it produces on the joint $i$ a torque $\tau_{i}=f_{r j} h$. We denote with $\delta_{i}$ the deflection of the elastic element realising the joint, that, according to beam theory, can be evaluated as

$$
\delta_{i}=\frac{-f_{r j} h l_{i}^{2}}{2 E_{i} I_{i}}
$$

where $E_{i}$ is the Young's modulus of the material, $I_{i}$ is the second moment of area, and $l_{i}$ is the length of the module elastic part. The corresponding joint rotation angle can be evaluated as

$$
\theta_{i}=\frac{-f_{r j} h l_{i}}{E_{i} I_{i}} .
$$

For the sake of simplicity we neglect the bending properties of the joint in the lateral and torsional direction.

The rotational stiffness of the joint can be then evaluated as

$$
k_{i}=\frac{E_{i} I_{i}}{l_{i}},
$$

Assuming both parts of the module as filled rectangular shape whose centroid is located at the origin, the second moment of area is given by

$$
I_{i}=\frac{w_{i} t_{i}^{3}}{12} .
$$

Referring to Eq. (11), we can observe that different joints stiffness can be achieved by changing either the geometric or material parameters of the modules. Since we aim at realising a modular structure, we assume that the geometric parameters are the same for each module, i.e. $I_{i}=I, l_{i}=l, h_{i}=h$. On the other hand, we can tune the material parameters in order to vary the modules stiffness. In particular, by keeping in view a possible $3 \mathrm{D}$ printing fabrication method, we can print the flexible parts with different percentage of infill density to get different stiffness values, while maintaining the same geometric shape of the parts. As an example, Table I summarizes the variation of Young's modulus $E$ of the material for the Thermoplastic Polyurethane as a function of the infill percentage density $\rho$ [14].

For a given geometric structure of the joint, using as possible realization technique the 3D printing with Thermoplastic Polyurethane, the corresponding stiffness value is therefore depending on the infill density percentage, higher densities correspond to higher stiffness values and vice-versa,

$$
k_{i}=f\left(\rho_{i}\right) .
$$

As an example, we evaluated with the Finite Element Method (FEM) based software COMSOL the stiffness of a modular element varying material properties. The dimensions of the elements are: length $l=13 \mathrm{~mm}$, thickness $t=$ $2.5 \mathrm{~mm}$, width $w=21 \mathrm{~mm}$. Fig. 1 shows the main features of the model, while Tab. I summarizes the obtained results, in terms of joint stiffness for different values of infill density
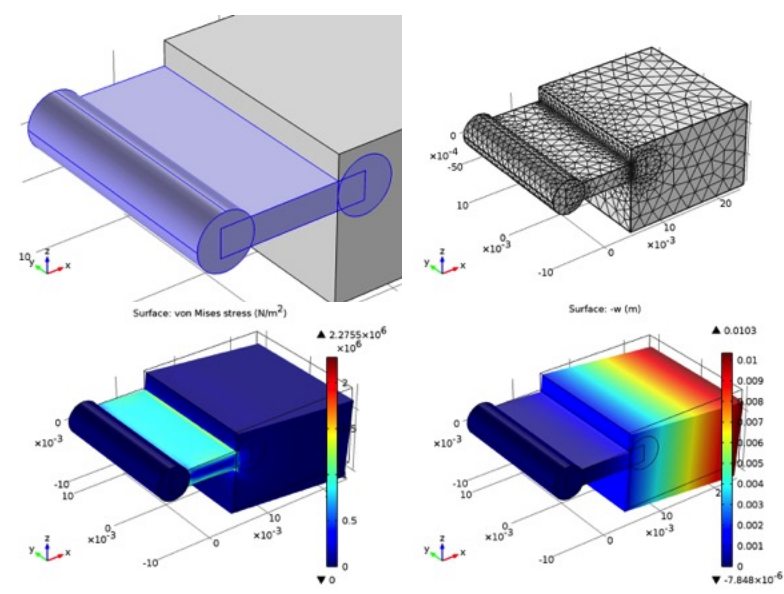

Fig. 1. FEM model and stiffness analysis of the passive joint.

TABLE I

TPU MECHANICAL PROPERTIES AS A FUNCTION OF 3D PRINTING INFILL DENSITY PERCENTAGE, FROM [14] AND RESULTS FROM FEM ANALYSIS OF THE PASSIVE JOINT, STIFFNESS VALUE AS A FUNCTION OF INFILL DENSITY PERCENTAGE OF THE MATERIAL, FOR A GIVEN GEOMETRY.

\begin{tabular}{|c|c|c|c|c|}
\hline$\rho \%$ & $\mathrm{E}(\mathrm{MPa})$ & $k(\rho) \mathrm{Nmm} / \mathrm{rad}$ & $k_{l} \mathrm{Nmm} / \mathrm{rad}$ & $k_{t} \mathrm{Nmm} / \mathrm{rad}$ \\
\hline \hline 10 & 1.07 & 4.6930 & 331.3 & 24.9 \\
\hline 30 & 1.38 & 6.0526 & 427.2 & 32.1 \\
\hline 50 & 2.07 & 9.0789 & 640.8 & 48.1 \\
\hline 70 & 6.53 & 28.6403 & 2021.6 & 151.7 \\
\hline 90 & 9.45 & 41.4474 & 2925.6 & 219.7 \\
\hline 100 & 10.50 & 46.052 & 3807.9 & 285.7 \\
\hline
\end{tabular}

percentage. We evaluated also the stiffness in the lateral direction, $k_{l}$, and the torsional stiffness, $k_{t}$. It is worth to observe that the lateral stiffness is much higher than the bending one, due to the module geometry, while the torsional one is closer.

\section{Actuator force evaluation}

The mathematical model can be extended to any number of modules to achieve desired length of the finger and local deflection of each module can be transformed to global frame. Let us consider a point $p_{i}\left(\delta_{i}, l_{i}\right)$ that describes the position and orientation of the module $i$, where $\delta_{i}$ denotes the local deflection between the part $i$ and $i-1$ and $l_{i}$ is the length of the part according to the notation introduced in [15].

In order to find the global coordinates of each module, we need to add the contributions from previous relative deflections and account for rotation due to the angle of the previous module. The standard homogenous transformation matrices (Eq. 8) can be used to perform this operation.

The model proposed above can be further exploited to relate the fingertip force to the actuator force. The force applied by the actuator through the tendon produces a moment about the flexible part of the finger. The resultant behavior can be approximated by a simplified cantilever beam model. The two forces acting on the model are the actuator applied force and the resultant reaction force which 
is equal to the fingertip applied force $F_{t i p}$. We can separately study the deflection effects generated by the two forces by using the superposition principle. Let $\delta_{a}$ be the deflection due to the actuator applied force and $\delta_{r}$ be the deflection due to the reaction force

$$
\delta_{a}=\frac{f_{r} h l^{2}}{2 E I}, \delta_{r}=\frac{F_{t i p} l^{3}}{3 E I} .
$$

The sum of both deflections can be equated to zero and the resultant equation is solved for $F_{t i p}$ as

$$
F_{\text {tip }}=\frac{3 f_{r} h}{2 l} \text {. }
$$

Note that the terms $E$ and $I$ are canceled out of the equation and as a result we do not need to consider the interaction between the alternating stiff and flexible parts of the modules to obtain the overall load at the fingertip. The model can be extended to any number of modules, as

$$
F_{t i p}=\frac{3 f_{r} h}{2 \sum_{i=1}^{2 N} l_{i}} .
$$

Furthermore, we can consider the parasitic capstan effects that takes place between the cable and stiff parts as the robotic finger transforms to a curve shape. As the modules lose the colinearity during their motion, the cable imparts a reaction force that resists further actuation. We can include the capstan effect by considering the angle between subsequent stiff parts of modules $\left(\theta_{i-1}-\theta_{i}\right)$. The tendon does not pass through the flexible parts, so we can only consider the parasitic capstan effects on stiff parts. Thus, the fingertip force can be modified as

$$
F_{\text {tip }}=\frac{3 f_{r} h}{2 \sum_{i=1}^{2 N} l_{i}} \prod_{i=2, \text { even }}^{2 n-2} e^{\mu\left(\theta_{i-1}-\theta_{i}\right)},
$$

where $\mu$ is the friction coefficient.

\section{CASE Study: The Soft-SiXthFinger}

The mathematical framework presented in Sec. II can be applied to a wide range of robotic grippers. In this section, we propose as a case study the Soft-SixthFinger. The SoftSixthFinger is a device designed to be used to compensate the missing grasping abilities of chronic stroke patients [12]. The exploded view of the device and its possible applications are shown in Fig. 2. The working principle of the device is to replicate the two parts of a simple gripper using on one side the paretic forearm of a patient and, on the other side, a flexible finger that can be worn at the wrist with the help of an elastic band. We use this device as case study for two main reasons. Firstly, in earlier version of the device [12], the trajectory of the finger was regulated manually by setting the joints stiffness by hit and trial methods. The proposed mathematical model framework allows to obtain suitable stiffness at each joint which improved not only the grasping performance of the device but also considerable reduce the manufacturing iterations. Secondly, the proposed method can be validated on a finger base. In fact, the idea is that a finger is able to track a certain trajectory when selecting

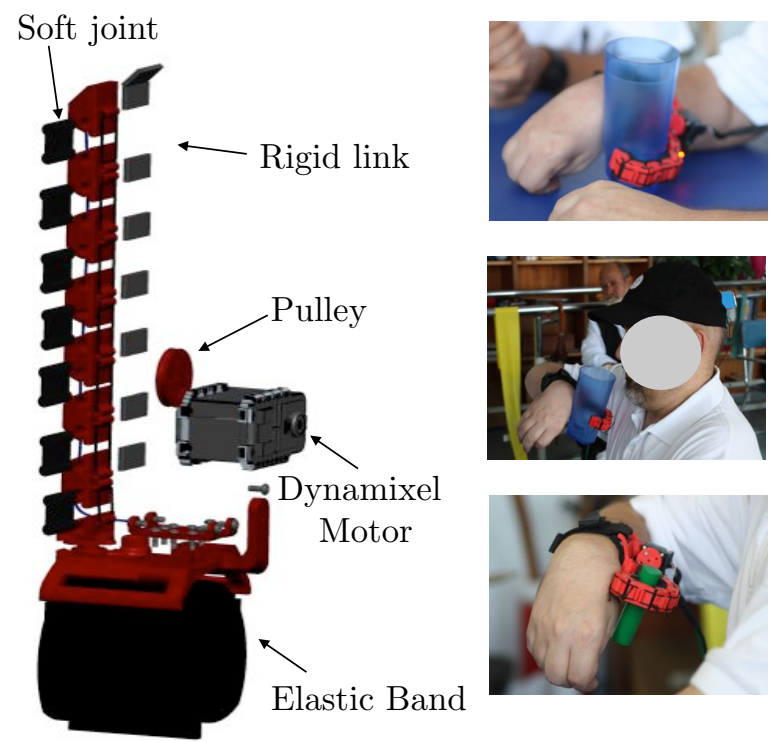

Fig. 2. The Soft-SixthFinger: The CAD exploded view of complete SoftSixthFinger and its working principle with paretic hand to compensate its missing abilities.
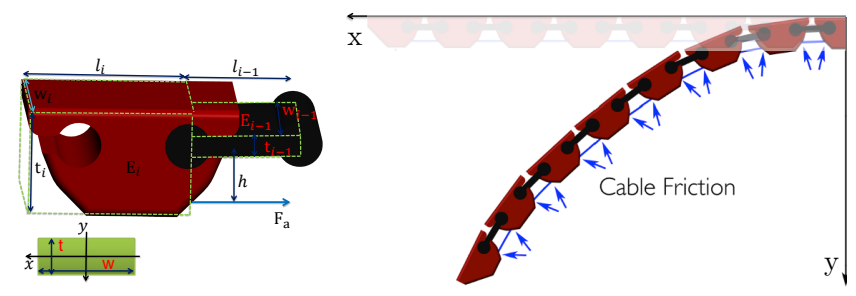

Fig. 3. On left, single module geometric and material parameters are shown. The module consists of flexible part $(i-1)$ and stiff part $(i)$. On right, complete cable driven Soft-SixthFinger with seven modules is shown. The global coordinates are defined at the base module.

the appropriate joint stiffness. In case of multi-fingered hand the process is simply replicated for each finger.

The Soft-SixthFinger is built with a modular structure. Each module is composed of a rigid 3D printed part realized in ABS (Acrylonitrile Butadiene Styrene, ABSPlus, Stratasys, USA) and a 3D printed thermoplastic polyurethane part (Lulzbot, USA) that acts as the flexible joint. We selected polyurethane for flexible parts because the high elongation of this material allows for repeated movement and impact without wear or cracking proving also an excellent vibration reduction. The geometric and material parameters for the unit module of Soft-SixthFinger are shown in the left side of Fig. 3 and their numerical values are same as defined in Section II-C. We considered seven modules in order to achieve a length of the finger similar to the average size of human hand [16]. The right side of Fig. 3 shows the complete cable driven flexible finger with seven modules where global coordinates are defined at the base module.

\section{A. Simulations}

To let the Soft-SixthFinger be able to grasp a wide range of objects, it is important to define a suitable finger flexion trajectory. To this aim, we took inspiration from the motion 


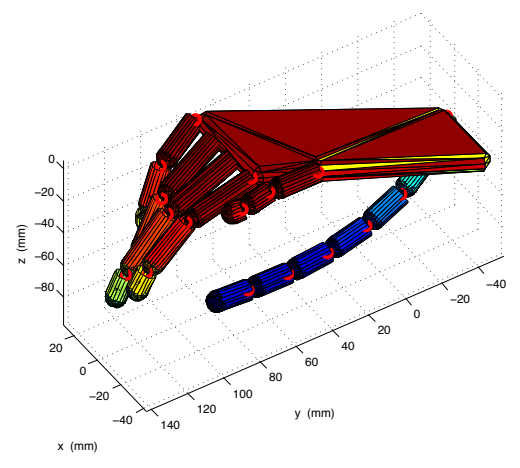

Fig. 4. The SynGrasp model of the human hand wearing the SoftSixthFinger used to compute the joint stiffness values.

of the human hand. In particular, we used the mapping algorithm originally proposed in [17], [18] to transfer the motion of the human hand onto robotic hands with dissimilar kinematics. The mapping method allows to replicate on the Soft-SixthFinger, the effects in terms of motions and deformations that a human reference hand would perform on a virtual object. This allows to work directly on the task space avoiding a specific projection between different kinematics. Details of the mapping are not reported here for the sake of brevity, interested reader can refer to [17], [18]. We considered a model of a human hand augmented with a model of the robotic finger, see Fig. 4. We simulated using the Matlab SynGrasp toolbox [19] the motion of the human hand according to the first synergy as defined in [20]. We then computed the trajectory of the robotic fingertip using the mapping algorithm. This target trajectory in the Cartesian space is the result of the mapping of the first human hand synergy onto the robotic device.

Once a target trajectory is defined, we take advantage of the stiffness design presented in Sec. II to simulate a tendon driven underactuated finger able to track the desired fingertip trajectory.

Considering Thermoplastic Polyurethane as material for the flexible joints, we computed the values for $\mathbf{k}_{q}$ using the method proposed in Sec. II. The obtained results are reported in Fig. 5. We then considered the mean values for $\mathbf{k}_{q}$ since we noticed that the stiffness variation for each joint for the proposed trajectory is low. More complex trajectories could results in stiffness values that cannot be approximated with the mean value. However, meaningful flexion trajectories for grasping can be well approximated. The stiffness values used were $6.6 \mathrm{Nmm} / \mathrm{rad}, 7.7 \mathrm{Nmm} / \mathrm{rad}, 9.2 \mathrm{Nmm} / \mathrm{rad}, 11.5$ $\mathrm{Nmm} / \mathrm{rad}, 15.3 \mathrm{Nmm} / \mathrm{rad}, 23.1 \mathrm{Nmm} / \mathrm{rad}, 46.1 \mathrm{Nmm} / \mathrm{rad}$ from joint one to seven, respectively, which correspond to the following infill density percentage 14.3, 16.7, 20, 25, $33.4,50,100$. The range of stiffness values for the joints was decided according to the properties of the material used for the soft joints. We selected the range of values that were possible to replicate using that material and a $3 \mathrm{D}$ printer.

Once we have the target trajectory and the relative values of joint stiffness, we can use the proposed numerical model to study the kinematics of the tendon-driven Soft-SixthFinger and to simulate its fingertip trajectory. We simulated the

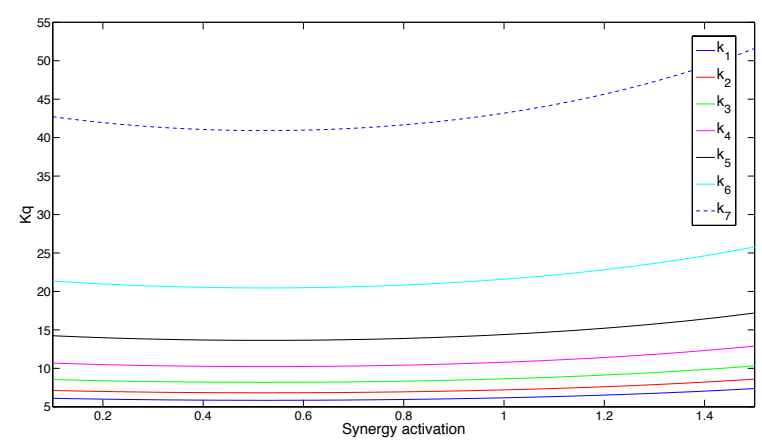

Fig. 5. Joint stiffness values necessary to generate the desired trajectory of the Soft-SixthFinger, evaluated according to Eq. (5), as a function of the corresponding actuation rate of the first synergy.
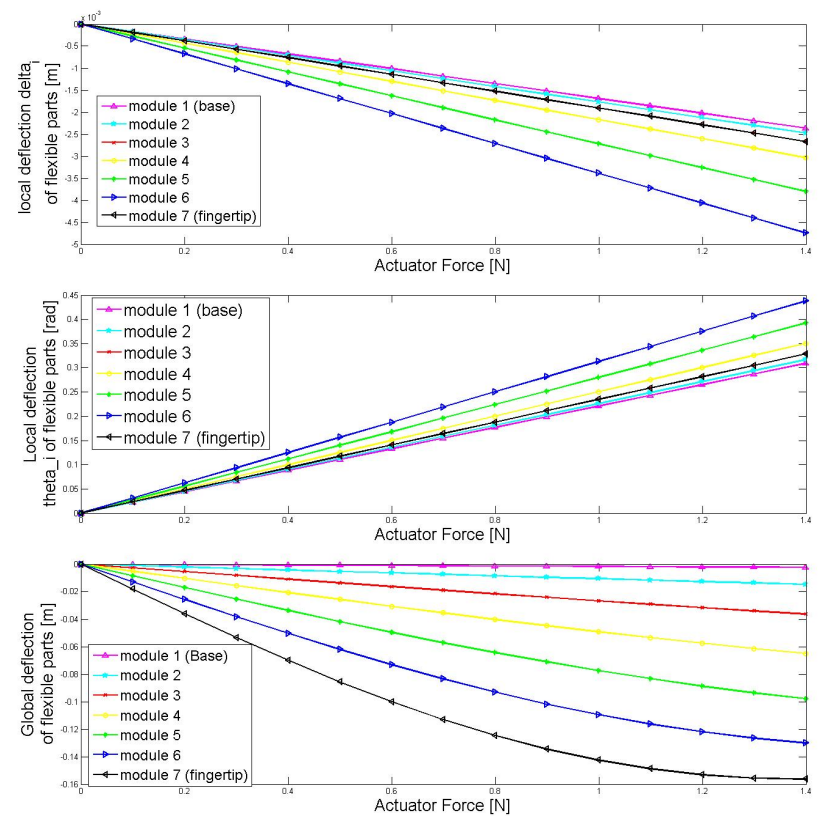

Fig. 6. The simulation results: local bending $\delta_{i}$ in each module vs. actuator force (top), local theta $\theta_{i}$ for each module vs. actuator force (middle) and overall bending of each module with respect to the base module vs. actuator force (bottom), are shown.

model with the infill density percentage reported.

The simulation results corresponding to the computed stiffness values are plotted in Fig. 6 and Fig. 7. In particular, Fig. 6 shows the bending deflection $\left(\delta_{i}\right)$ and the angle $\left(\theta_{i}\right)$ for each module with respect to its local coordinates and the overall deflection of each joint with respect to global coordinates defined at the base of the finger. Fig. 7 shows the plot of the actuator applied force to fingertip force.

\section{B. Experiments}

In order to validate our method, as well as to observe stiffness profile via an empirical comparative study, we realized two Soft-SixthFinger configurations with different stiffness at joint level. We 3D printed the flexible parts with different percentage of infill density to achieve different deflection in joints under same applied tendon force. A healthy subject worn the devices on its right arm while 


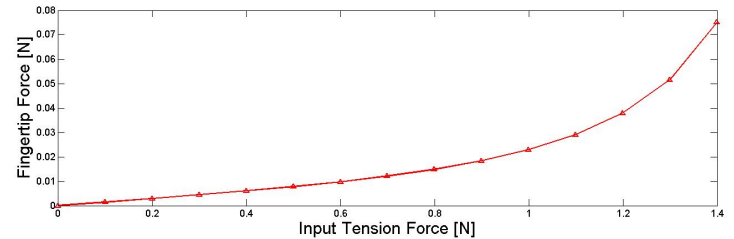

Fig. 7. Simulation results of the actuator force $\left(f_{r}\right)$ versus Fingertip Force $\left(F_{t i p}\right)$.

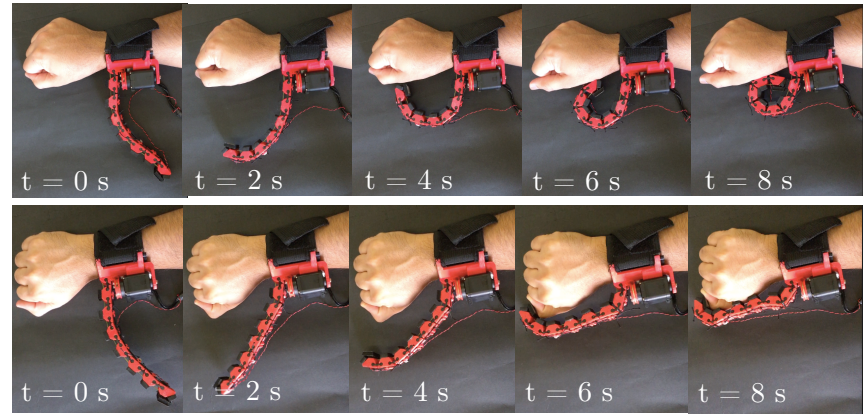

Fig. 8. Trajectories of the Soft-SixthFinger using different stiffeness at joint level. On top the joint stiffness is computed using the design method introduced in this paper. On the bottom, the trajectory obtained considering all the joints with the same stiffness value.

simulating the paretic hand. The aim of the experiment was to verify how the joints' stiffness regulate the trajectory of the finger and, consequently, how the flexion trajectory influence the device adaptability to different shapes of the grasped objects.

Fig. 8 shows the snapshots of two configurations of SoftSixthFinger with different joints stiffness. The top configuration has the stiffness in each joint computed using the design method introduced in this paper. The the bottom one shows the configuration with the same stiffness in all the joints.

\section{Conclusions}

In this paper, we presented an approach to model the kinematics and the passive stiffness of flexible grippers and its realization in order to follow a desired trajectory for the fingertip. We explored the role of stiffness in grasping performance and demonstrated a novel method which is applicable to any tendon-driven, underactuated and passively compliant hand. The grasping performance of such grippers mainly depends on their intrinsic characteristics, e.g., passive joint compliance, instead of relying on active control for compliance used in complex manipulators. We defined firstly a procedure to determine suitable joints stiffness and then we proposed a possible realization in robotic finger prototype. Without any loss of generality, we proposed a modular approach to define robotic hands. The feasibility of the approach was demonstrated through a framework composed of both simulations and exploitation of model in the realization of the Soft-SixthFinger.

Currently, we are working on embedding the presented deflection based beam theory for soft hands in the SynGrasp Matlab toolbox. We are also working on the realization of multi-fingered grippers based on the same design principles of the Soft-SixthFinger.

\section{REFERENCES}

[1] D. Prattichizzo, M. Malvezzi, M. Gabiccini, and A. Bicchi, "On motion and force controllability of precision grasps with hands actuated by soft synergies," IEEE Transactions on Robotics, vol. in press, pp. 117,2013

[2] A. T. Miller, S. Knoop, H. I. Christensen, and P. K. Allen, "Automatic grasp planning using shape primitives," in Robotics and Automation, 2003. Proceedings. ICRA'03. IEEE International Conference on vol. 2, pp. 1824-1829, IEEE, 2003.

[3] L. Birglen, T. Lalibertè, and C. Gosselin, Underactuated Robotic Hands, vol. 40 of Springer Tracts in Advanced Robotics. Springer, 2008.

[4] A. M. Dollar and R. D. Howe, "The highly adaptive sdm hand: Design and performance evaluation," The international journal of robotics research, vol. 29, no. 5, pp. 585-597, 2010.

[5] M. G. Catalano, G. Grioli, E. Farnioli, A. Serio, C. Piazza, and A. Bicchi, "Adaptive synergies for the design and control of the pisa/iit softhand," The International Journal of Robotics Research, vol. 33, no. 5, pp. 768-782, 2014.

[6] R. Deimel, C. Eppner, J. Alvarez-Ruiz, M. Maertens, and O. Brock, "Exploitation of environmental constraints in human and robotic grasping," in International Symposium on Robotic Research, 2013.

[7] R. Deimel and O. Brock, "A compliant hand based on a novel pneumatic actuator," in Robotics and Automation (ICRA), 2013 IEEE International Conference on, pp. 2047-2053, IEEE, 2013.

[8] L. U. Odhner, L. P. Jentoft, M. R. Claffee, N. Corson, Y. Tenzer, R. R. Ma, M. Buehler, R. Kohout, R. D. Howe, and A. M. Dollar, "A compliant, underactuated hand for robust manipulation," The International Journal of Robotics Research, vol. 33, no. 5, pp. 736-752, 2014.

[9] M. Liarokapis and A. M. Dollar, "Post-contact, in-hand object motion compensation for compliant and underactuated hands," in IEEE International Symposium on Robot and Human Interactive Communication, 2016.

[10] J. Butterfass, M. Grebenstein, H. Liu, and G. Hirzinger, "DLR-hand II: next generation of a dextrous robot hand," in Robotics and Automation, 2001. Proceedings 2001 ICRA. IEEE International Conference on, vol. 1, pp. 109-114, 2001

[11] F. Sanfilippo, G. Salvietti, H. Zhang, H. P. Hildre, and D. Prattichizzo, "Efficient modular grasping: an iterative approach," in Proc. IEEE Int Conf. on Biomedical Robotics and Biomechatronics, (Rome, Italy), pp. 1281-1286, 2012

[12] I. Hussain, G. Salvietti, G. Spagnoletti, and D. Prattichizzo, "The soft-sixthfinger: a wearable emg controlled robotic extra-finger for grasp compensation in chronic stroke patients," IEEE Robotics and Automation Letters, vol. 1, pp. 1000 -1006, July 2016.

[13] D. Wren and R. B. Fisher, "Dextrous hand grasping strategies using preshapes and digit trajectories," in Systems, Man and Cybernetics, 1995. Intelligent Systems for the 21st Century., IEEE International Conference on, vol. 1, pp. 910-915, IEEE, 1995.

[14] J. A. Gopi and G. B. Nando, "Modeling of youngs modulus of thermoplastic polyurethane and polydimethylsiloxane rubber blends based on phase morphology," Advances in Polymer Science and Technology: An International Journal, pp. 43-51, 2014.

[15] S. Timoshenko and J. Gere, Mechanics of materials. Van Nostrand Reinhold Co., 1972.

[16] R. J. Schwarz, "The anatomy and mechanics of the human hand," Artificial Limbs, vol. 22, 1955.

[17] G. Gioioso, G. Salvietti, M. Malvezzi, and D. Prattichizzo, "Mapping synergies from human to robotic hands with dissimilar kinematics: an approach in the object domain," IEEE Trans. on Robotics, 2013.

[18] D. Prattichizzo, G. Salvietti, F. Chinello, and M. Malvezzi, "An objectbased mapping algorithm to control wearable robotic extra-fingers," in Proc. IEEE/ASME Int. Conf. on Advanced Intelligent Mechatronics, (Besanon, France), pp. 1563-1568, 2014.

[19] M. Malvezzi, G. Gioioso, G. Salvietti, and D. Prattichizzo, "Syngrasp: A matlab toolbox for underactuated and compliant hands," Robotics Automation Magazine, IEEE, vol. 22, pp. 52-68, December 2015.

[20] M. Santello, M. Flanders, and J. F. Soechting, "Postural hand synergies for tool use," The Journal of Neuroscience, vol. 18, pp. 10105-10115, December 1998. 